\title{
Decomposition of carboxymethyl cellulose based on nano-knife principle
}

Qin Zhou ${ }^{1}$, Li Hong ${ }^{1}$, Marcello Di Bonito ${ }^{2}$, Gang Pan ${ }^{1,2, *}$

1. Key Laboratory of Environmental Nanotechnology and Health Effects, Department of Environmental Nano-materials, Research Center for Eco-Environmental Sciences, Chinese Academy of Sciences, Beijing 100085, China. E-mail: qinzhou@rcees.ac.cn

2. Center of Integrated Water-Energy-Food studies (iWEF), Nottingham Trent University, Nottinghamshire NG250QF, UK

\begin{abstract}
The traditional degradation of organic pollutants is based on the sacrifice of chemical or biological reagents. In this study, a purely physical technique was developed to break the chemical bonds and consequently decompose macromolecules in aqueous solution. Assisted with a high-speed mechanical blade, refined quartz sand grains with particularly sharp nano-scale edges can act as 'nano-knives', which are able to cut the long chain of carboxymethyl cellulose (CMC, as a model molecule). High performance size exclusion chromatography measurements evidenced that the original CMC molecules (41000 Da) were decomposed into a series of smaller molecules $(460,1000,2200,21,000,27,000$ and 31,000 Da). Consequently, the initial viscosity of the CMC solution $(2 \mathrm{~g} / \mathrm{L})$ rapidly decreased by approximately $50 \%$ after 3 min treatment by the nano-knife materials along with the mechanical blade. Fourier transform infrared (FTIR) spectra indicated that the original functional groups were
\end{abstract}


still present and new functional groups were not produced after shearing. The intensity of the main functional group $\beta$-1-4-glycosidic bond (wavenumber $1062 \mathrm{~cm}^{-1}$ ) was observed to markedly decrease after shearing. These results indicated that the long-chain $\mathrm{CMC}$ was cleaved into short-chain CMC. A degradation mechanism was proposed whereby the cutting force generated by the rapid motion of the nano-knives may be responsible for the breakage of $\beta$-1-4-glycosidic bonds in the macromolecular cellulose backbone. These results provide support for a potentially more affordable and environment-friendly strategy for physical-based decomposition of recalcitrant organic pollutants from aqueous solution without the need of chemical or biological reagents.

\section{Keywords:}

Nano-knives

Shear degradation

Carboxymethyl cellulose

Glycosidic bond

Physical-based decomposition

* Corresponding author. E-mail: gpan@rcees.ac.cn (Gang Pan)

\section{Introduction}

The current degradation techniques for the majority of organic pollutants include mainly catalytic (Li et al., 2015), ultrasonic (Cheng et al., 2010; Mohod and Gogate, 
2011; Taghizadeh et al., 2012), thermal (de Britto and Assis, 2009; Zhang et al., 2016), radiant (Choi et al., 2008), electrochemical (Yang et al., 2015) and biological degradation (Gao et al., 2014) depending on the properties of the organic compounds. Atoms are taken away from the molecules and recombined to become new substances based on chemical reagents or additional energy. All of these approaches have been applied with some degree of success, but present some issues. The chemical methods sacrifice the reagents and may cause secondary environmental pollution. Biological technology is benign but needs more time to achieve full mineralization.

Generally, chemical or biological reactions can break down chemical bonds and degrade the pollutants under some specific conditions. However, many people have a dream of performing degradation without using chemical or biological reagents, so as to no longer have issues of pollution, toxicity, and so on. What will happen if a purely physical principle can be used to decompose molecules? That has never been possible before, especially in aqueous solution. However, it is not impossible because people currently use a mechanical method (ball milling) to break down molecules (Zhang et al., 2011, 2010). However, the molecules are in the solid phase and the method will release some amount of heat, which results in thermal pollution. This mechanical application is invalid for contaminants in aqueous solution.

Could it be possible to use a knife to cut a chemical bond? It is very hard to understand internal bonding interactions. However, our experiment has demonstrated that perhaps this is possible for certain kinds of chemical bonds. First of all, the knife must be very sharp and the size must match the chemical bond. 
In this study, the compound carboxymethyl cellulose $\left(\mathrm{CMC},\left(\mathrm{C}_{6} \mathrm{H}_{9} \mathrm{O}_{4}-\mathrm{OR}\right)_{n}\right)$, which is a synthetic polymer compound and widely used in various manufacturing processes for cosmetics, food, medicines and paper making (Bansal et al., 2010; Zorba and Ova, 1999), was chosen as a model macromolecule. Refined quartz sand grains with particularly sharp nano-scale edges act as nano-knives when they rotate under the hydrodynamic force generated by a high-speed mechanical blade in the presence of water. The influence of quartz sand mass and shearing time on CMC solution viscosity, the molecular size distribution and weight-average molecular weight (WAMW) were also investigated. The results indicated that this kind of molecule can be cut into smaller ones. The solution viscosity also showed a dramatic change. The main aim of the study is to test the development of a technique that could deal with the disposal of environmental pollutants from aqueous solution, such as CMC, without the use of chemical or biological agents but only relying on physical degradation.

\section{Materials and methods}

\subsection{Materials and analytical reagents}

Carboxymethyl cellulose (CMC), disodium phosphate dodecahydrate $\left(\mathrm{Na}_{2} \mathrm{HPO}_{4} \cdot 12 \mathrm{H}_{2} \mathrm{O}\right.$, analytical reagent (AR)), $\mathrm{NaH}_{2} \mathrm{PO}_{4} \cdot 2 \mathrm{H}_{2} \mathrm{O}$ (sodium dihydrogen phosphate dihydrate, $\mathrm{AR}$ ), $\mathrm{NaCl}$ (sodium chloride, $\mathrm{AR}$ ) and potassium bromide ( $\mathrm{KBr}$, spectrum pure (SP)) were obtained from Sinopharm Chemical Reagent Co., Ltd. (China). Quartz sand (2000 mesh, $6.5 \mu \mathrm{m}$ ) as nano-knife material was obtained from the Hebei Province, China. Sodium polystyrene sulfonate (PSS) with different 
molecular weights was purchased from Sigma-Aldrich, USA. All the chemicals were analytical reagent grade and used without further purification. Deionized water from a Millipore Milli-Q system (18 M $/ \mathrm{cm})$ was used for all experiments.

\subsection{Shearing experiment}

Batches of a known volume $(150 \mathrm{~mL})$ of CMC solution were prepared with different amounts of quartz sand $(1,2$ or $3 \mathrm{~g})$ and added into the container of a commercial blender with high-speed shearing blade (KS-995, KPS Co., China). The blender was then run at a speed of $18,000 \mathrm{r} / \mathrm{min}$ for $3 \mathrm{~min}$, with a $1 \mathrm{~min}$ rest every min of rotation to minimize the increase of temperature. The supernatant solution was collected and filtered through a $0.22 \mu \mathrm{m}$ membrane after centrifuging at $9000 \mathrm{r} / \mathrm{min}$ for $30 \mathrm{~min}$. The viscosity of CMC solutions was determined at room temperature by a rotary viscometer (NDJ-1, Shanghai Yueping scientific instrument Co., China). The size distribution and the weight-average molecular weight (WAMW) of CMC were determined by high performance size exclusion chromatography (HPSEC, Waters Co., USA) at room temperature. The blank control experiment was carried with the same parameters but without quartz sand. Each shearing experiment was carried out in duplicate.

\subsection{HPSEC measurements}

HPSEC was used to determine the size distribution and the WAMW of CMC before and after shearing. The HPSEC system was equipped with a double wavelength detector (Waters 2487, Waters Co., USA) and a pump (Waters 1525, Waters Co., USA). The chromatographic column was constituted of TSK-GEL 
G3000SWXL $(5 \mu \mathrm{m}, 7.8 \mathrm{~mm} \times 300 \mathrm{~mm})$. The mobile phase consisted of $0.2 \mathrm{~mol} / \mathrm{L}$ phosphate buffer and $0.5 \mathrm{~mol} / \mathrm{L} \mathrm{NaCl}$ solution at a flow rate of $0.8 \mathrm{~mL} / \mathrm{min}$ at room temperature (Chen et al., 2016). The preparation of the mobile phase was as follows: $7.1628 \mathrm{~g} \mathrm{Na}_{2} \mathrm{HPO}_{4} \cdot 12 \mathrm{H}_{2} \mathrm{O}, 3.1202 \mathrm{~g} \mathrm{NaH} \mathrm{PO}_{4} \cdot 2 \mathrm{H}_{2} \mathrm{O}$ and $3.1202 \mathrm{~g} \mathrm{NaCl}$ were dissolved into $100 \mathrm{~mL}$ Milli Q water. Then, $12.25 \mathrm{~mL} \mathrm{Na} \mathrm{HPO}_{4} \cdot 12 \mathrm{H}_{2} \mathrm{O}, 12.75 \mathrm{~mL}$ $\mathrm{NaH}_{2} \mathrm{PO}_{4} \cdot 2 \mathrm{H}_{2} \mathrm{O}$ and $20 \mathrm{~mL} \mathrm{NaCl}$ were mixed and diluted to $1 \mathrm{~L}$. The mobile phase was filtered through a $0.22 \mu \mathrm{m}$ membrane, and then degassed for $10 \mathrm{~min}$. The injection volume was $200 \mu \mathrm{L}$ and the total running time was $18 \mathrm{~min}$. The detection wavelength was set at $254 \mathrm{~nm}$ and the analysis was run in duplicate. The calibration was carried out using sodium polystyrene sulfonate (PSS) as standard, and the WAMW was calculated using Empower software (System Software, Empower option GPC, Waters Co., USA).

\subsection{FTIR measurements}

FTIR spectra of the samples were obtained on a FTIR spectrophotometer (NEXUS 670, Thermo Fisher, USA) by the KBr disk method. $2 \mathrm{mg}$ of the sample and $200 \mathrm{mg}$ of dried $\mathrm{KBr}$ was utilized for each measurement, and spectra were obtained in the wavelength region between 400 and $4000 \mathrm{~cm}^{-1}$. The acquired spectra were the result of 16 scans at a spectrophotometer resolution of $4.0 \mathrm{~cm}^{-1}$.

\section{Results and discussion}

\subsection{HPSEC analysis and decomposition products}

To explore whether the $\mathrm{CMC}$ macromolecule was broken into smaller molecules, HPSEC was used to analyze the molecular weight and its distribution before and after 
shearing (Fig. 1).
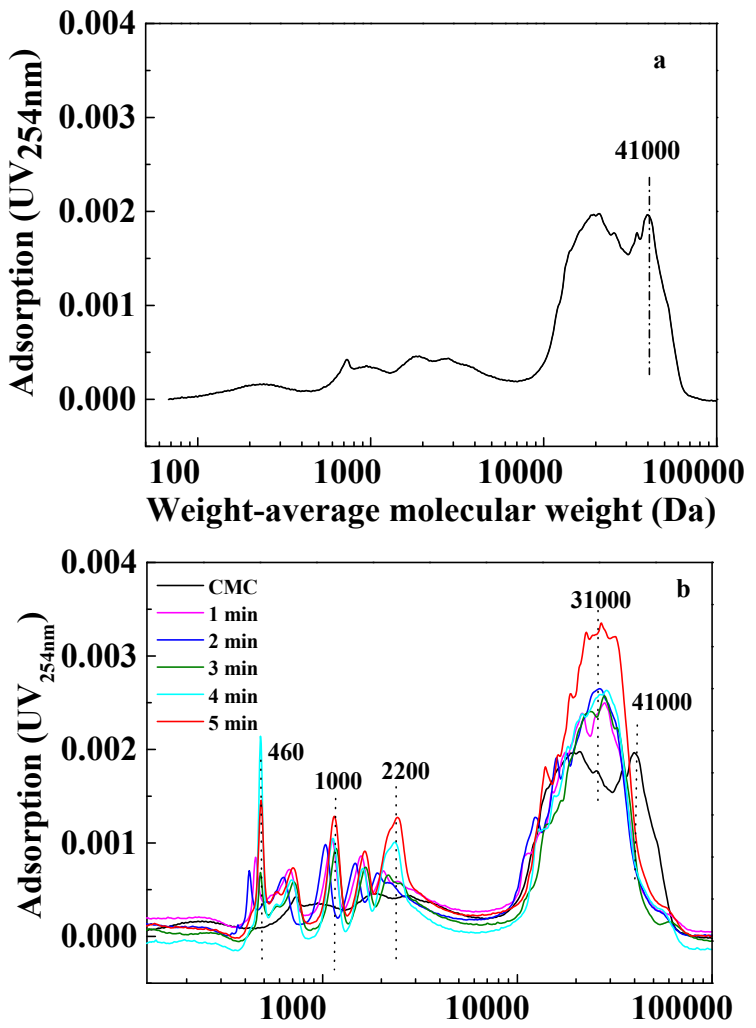

Weight-average molecular weight (Da)
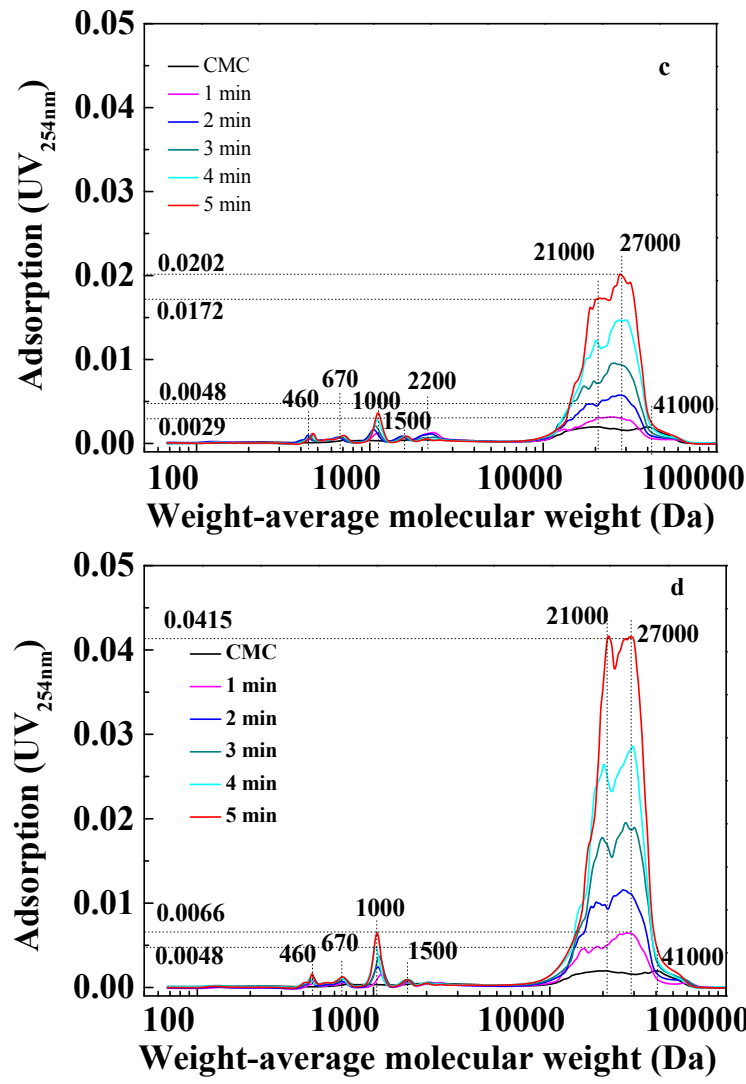

Fig. 1 Influence of work time and quartz sand mass on weight-average molecular 
weight of carboxymethyl cellulose (CMC). Conditions: initial concentration of CMC $C_{0 \text {-CMC }} 1 \mathrm{~g} / \mathrm{L}$. (a) original CMC, (b) quartz sand mass was $0 \mathrm{~g}$, (c) quartz sand mass was $1 \mathrm{~g}$, and (d) quartz sand mass was $3 \mathrm{~g}$.

The weight-average molecular weight (WAMW) of the original carboxymethyl cellulose was 41,000 Da (Fig. 1a). After high-speed shearing by the mechanical blade (Fig. 1b), the peak at 41,000 Da disappeared. At the same time, some new molecular weight peaks at 460, 1000, 2200 and 31,000 Da were observed. The result indicates that the initial macromolecule with 41,000 Da molecular weight (MW) was decomposed into smaller molecules by the mechanical blade. The absorbance of decomposition products was much lower, corresponding to the lower concentrations. Though the concentrations of smaller molecules were low, the decomposition of macromolecular CMC also resulted in the reduction of viscosity of the CMC solution from 8.9 to $7.4 \mathrm{mPa} \cdot \mathrm{sec}$ (shown in Fig. 2a).

The influence of work time and quartz sand mass on the WAMW of CMC is shown in Fig. 1c and d. When $1 \mathrm{~g}$ of quartz sand was added into the shearing system, the peak at 31,000 Da was replaced by two peaks at 21,000 and 27,000 Da (Fig. 1c). The small molecules with 460, 1000 and 2200 Da were still observed. Meanwhile, new molecular weight peaks at 670 and 1500 Da were detected. The absorbance of all decomposition products increased with the increase of shearing time from 1 to $5 \mathrm{~min}$. Specifically, the absorbance of 21,000 and 27,000 Da molecule increased from 0.0029 and 0.0048 to 0.0172 and 0.0202 , respectively, as the shear time increased from 1 to 5 min. This result implied that the amount of 21,000 and 27,000 Da molecules increased 
as the shear time increased.

Furthermore, when $3 \mathrm{~g}$ of quartz sand was used for 5 min work time, the absorbance of 21,000 and 27,000 Da molecules increased rapidly to 0.0415 , respectively (Fig. 1d). Meanwhile, the curve of the molecular weight distribution of CMC after shearing became narrower, indicating that the polydispersity became reduced (D'Almeida and Dias, 1997). The polydispersity means the inhomogeneity of the molecular weight. Fig. 1d shows that the reduction of polydispersity appears to be greater with increasing work time. In other words, the polydispersity or inhomogeneity is reduced because the degraded molecules enrich the low MW fraction. Degradation of the high MW chains generates molecules of smaller dimension, reducing both the number- and weight-average molecular weights. This reduction is greater as the fraction of molecules capable of being degraded increases.

The appearance of molecules with various WAMW indicates that the large molecules could be decomposed into smaller ones by the nano-knife material and that the content of smaller-molecular-weight compounds increased with the increase of nano knife amount and shear time.

\subsection{Viscosity measurements}

To some degree, the change of viscosity of CMC solution may also indirectly reflect the change in molecular weight. The viscosity of the CMC solution denotes the degree of polymerization at the same concentration and temperature. Polymer degradation is usually quantified either by molecular weight distribution or by change in the intrinsic viscosity (Mohod and Gogate, 2011). Generally, the higher the 
molecular weight, the higher the viscosity will be.

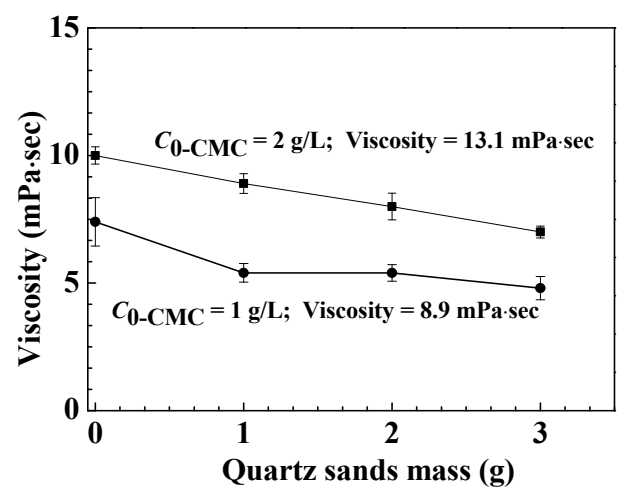

Fig. 2 Influence of quartz sand mass on viscosity of CMC solution with the shear time $3 \mathrm{~min}$.

The initial viscosities of carboxymethyl cellulose solutions were 8.9 and 13.1 $\mathrm{mPa} \cdot \mathrm{sec}$ with $\mathrm{CMC}$ concentrations of 1 and $2 \mathrm{~g} / \mathrm{L}$, respectively (Fig. 2). The viscosity was reduced to 7.4 and $10.0 \mathrm{mPa} \cdot \mathrm{sec}$, respectively, after $3 \mathrm{~min}$ as a result of the highspeed shearing of the machine (HSS-M) alone (no quartz sand added). This corresponds to reduction in the CMC viscosity of $16.9 \%$ and $23.7 \%$ (Fig. 2). An earlier study indicated that water turbulent flow was not able to shear the CMC molecules in aqueous solution (D'Almeida and Dias, 1997). Therefore, the reductions of CMC viscosity $(16.9 \%$ and $23.7 \%)$ were caused by the commercial blender with $18,000 \mathrm{r} / \mathrm{min}$ high-speed shearing force. When $3 \mathrm{~g}$ of quartz sand was added into the above systems, the viscosity was reduced to 4.8 and $7.0 \mathrm{mPa} \cdot \mathrm{sec}$, respectively, after shearing $3 \mathrm{~min}$, which corresponds to reductions in CMC viscosity of $46.1 \%$ and $46.6 \%$. In other words, the additional efficiency $(29.2 \%$ and $22.9 \%)$ can be attributed to the presence and action of the quartz sand. Another study reported that the viscosity of CMC solution decreased by $35 \%$ after 30 min by HSS-M when the shearing rate 
was $12,000 \mathrm{r} / \mathrm{min}$ (Li et al., 2011b). Results from the present study indicate that the introduction of quartz sand into the system can effectively shorten the time and enhance the decomposition efficiency. Furthermore, the viscosity of CMC decreased with increasing amounts of quartz sand (Fig. 2).

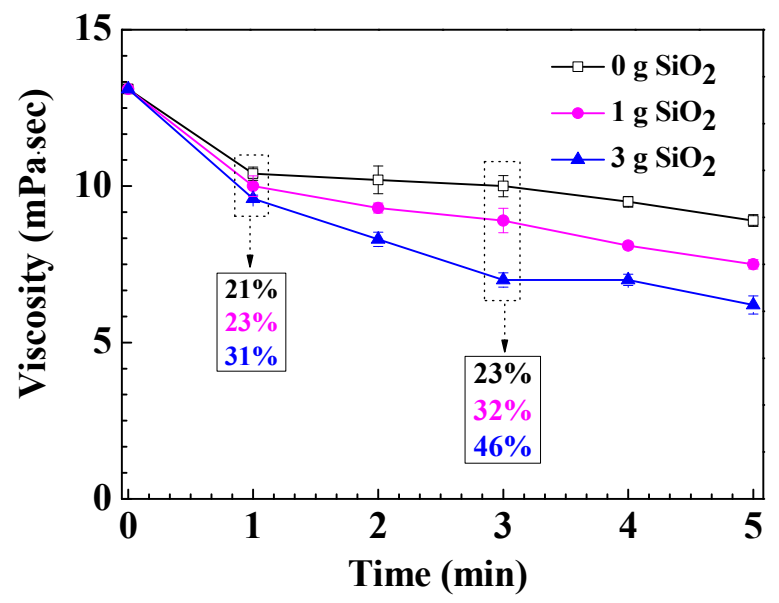

Fig. 3 Influence of work time on viscosity of CMC solution with $C_{0 \text {-CMC }} 2 \mathrm{~g} / \mathrm{L}$ and viscosity $13.1 \mathrm{mPa} \cdot \mathrm{sec}$.

The influence of shear time on the viscosity of CMC solutions (Fig. 3) was investigated. The CMC solution viscosity was significantly decreased by $21 \%$ within $1 \mathrm{~min}$ by the high-speed shearing action of the mechanical blade. At this specific time-point, the influence of the addition of quartz sand is less evident, with a difference of less than $10 \%$ between the results obtained with various amounts of quartz sand added.

When the shearing time increased from 1 to $3 \mathrm{~min}$, the reduction in viscosity related to HSS-M was much less significant (21\% vs. $23 \%)$. On the other hand, the reduction in viscosity measured when adding $3 \mathrm{~g}$ of quartz sand increased to up to 23\%. Following the findings in a previous report (Mohod and Gogate, 2011), the 
nano-knives seem to be effective in degrading CMC in the solution as shown by the reduction in viscosity.

\subsection{Decomposition mechanism}

Fig. 1b shows that use of the high-speed mechanical blade alone can cleave the macromolecule $\mathrm{CMC}$, but the concentration of the decomposition products was very low. Though the high-speed rotation of mechanical blade is able to produce a large shearing force, it is likely that the presence of water weakened the potential total output, with hydrodynamic force potentially originating as a result of accelerated moving water molecules (Mohod and Gogate, 2011). Therefore, as the bond energies of $\mathrm{C}_{1}-\mathrm{O}-\mathrm{C}_{4}$ and of $\mathrm{C}_{1}-\mathrm{O}-\mathrm{C}_{5}$ are reported to be 313 and $431 \mathrm{~kJ} / \mathrm{mol}$, respectively ( $\mathrm{Li}$ et al., 2011b), it is likely that the direct HSS-M acting force, added to the counteracting CMC viscosity or the hydrodynamic forces, may not have been enough to break down the long chain of the $\mathrm{CMC}$ molecule.

On the other hand, the solution viscosity decreased very significantly (more than $20 \%$ ) within 1 min without quartz sand. When the CMC was dissolved in the aqueous solution, intra-molecular and intermolecular hydrogen bonds formed and constituted a network thread structure (Cao et al., 1997). The energy generated from the high-speed rotation of a mechanical blade is greater than that of hydrogen bonds $(19-34 \mathrm{~kJ} / \mathrm{mol})$ (He et al., 2002), therefore allowing them to be broken and the network structure to be disaggregated, resulting in a reduction in degree of polymerization and a decrease in the CMC solution viscosity.

It is therefore likely that the main $\mathrm{CMC}$ molecular chain then became exposed 
after the network structure was disaggregated by hydrodynamic forces. When the quartz sand grains were added into the system, the frictional forces between these and CMC molecules must have increased as a result. The motion of the mechanical blade moved the quartz sand grains effectively and produced an overall physical mechanism, in which the nano-scale edges of the sand grains acted as the equivalent of sharp nano-knives. These movements are likely to set up large shear fields, splitting the most susceptible chemical bonds of $\mathrm{CMC}$ molecules. As a consequence, the distribution and the WAMW changed and the solution viscosity further decreased after shearing (Figs. 1 and 2). In general, it can therefore be concluded that the shearing force of the nano-knives does have a significant effect on the degradation of CMC molecules.

FTIR spectra of the CMC before and after shearing were measured to verify whether there were new degradation products with different functional groups or not (Fig. 4a). In agreement with previous studies (de Britto and Assis, 2009; Ruzene et al., 2007), typical absorption bands of the CMC FTIR spectrum were observed at 3400 $\mathrm{cm}^{-1}$ (axial deformation of $\mathrm{OH}$ ), $2900 \mathrm{~cm}^{-1}$ (axial deformation of $\mathrm{C}-\mathrm{H}, \mathrm{CH}_{2}$ ), $1595 \mathrm{~cm}^{-}$ ${ }^{1}\left(\mathrm{C}=\mathrm{C}\right.$ asymmetrical axial deformation), $1417 \mathrm{~cm}^{-1}$ (symmetrical axial deformation), and $1380 \mathrm{~cm}^{-1}$ (angular deformation on the plane of $\mathrm{OH}$ ). One of the absorption bands occurred also at $1062 \mathrm{~cm}^{-1}$, which was assigned to the stretching vibration of the $\beta-1$ 4-glycosidic bond $\left(\mathrm{C}_{1}-\mathrm{O}-\mathrm{C}_{4}\right)$ in $\mathrm{CMC}$.

Even though the intensities of the peaks observed were greater in the original sample of $\mathrm{CMC}$, the spectra of $\mathrm{CMC}$ after shearing presented similar transmittance 
values. The overall spectra pattern did not change after shear force by both the nanoknives and mechanical blade, and no additional bands appeared. This indicates that the important functional groups were still present and no new functional groups were produced after shearing. CMC molecules are rather strong because of the presence of benzene rings in the backbone, where the energy required to break them should reach 2076-2868 kJ/mol (D'Almeida and Dias, 1997). The long-chain CMC was only cleaved into short-chain $\mathrm{CMC}$, that is, $\mathrm{CMC}$ oligomers were obtained. Meanwhile, the schematic diagram of degradation of CMC is shown in Fig. $\mathbf{4 b .}$
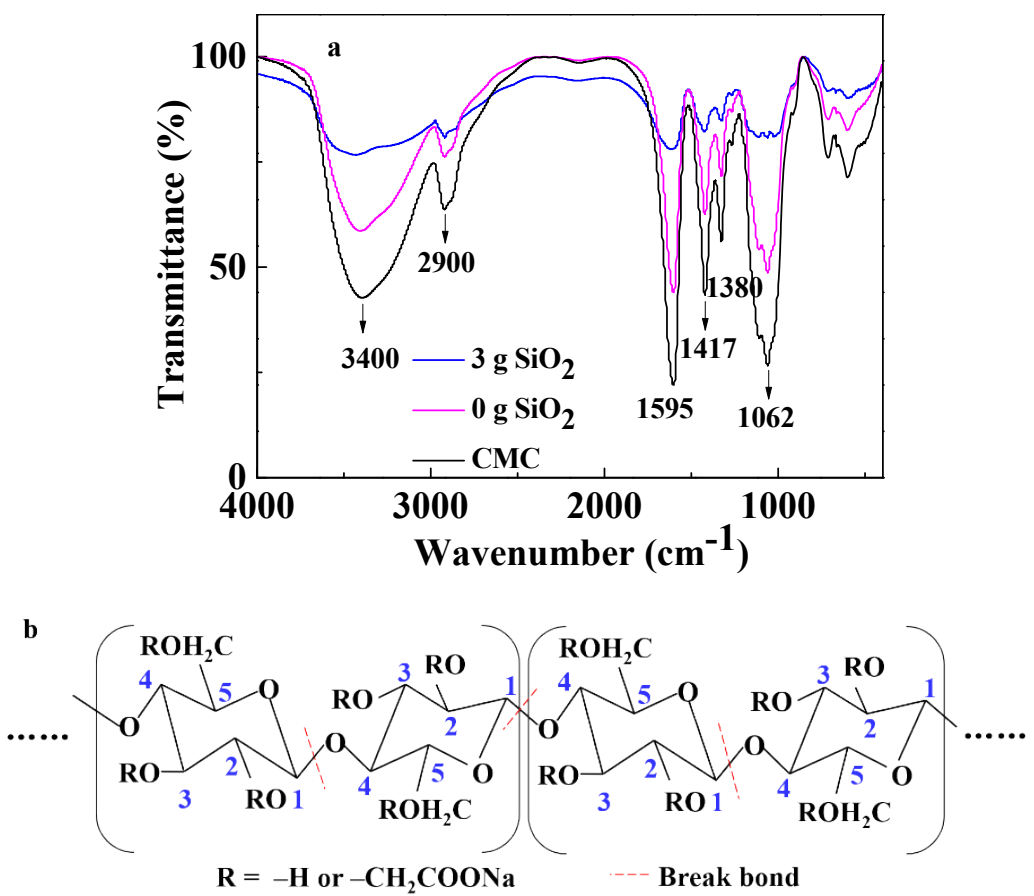

Fig. 4 (a) Fourier transform infrared (FTIR) spectra of the CMC before and after shearing with $C_{0-\mathrm{CMC}} 1 \mathrm{~g} / \mathrm{L}$ and work time $5 \mathrm{~min}$, and (b) schematic diagram of degradation of CMC.

The intensity observed at $1062 \mathrm{~cm}^{-1}$ was assigned to the stretching vibration of $\beta$ 1-4-glycosidic bonds $\left(\mathrm{C}_{1}-\mathrm{O}-\mathrm{C}_{4}\right)$ in $\mathrm{CMC}$, and was markedly decreased after shearing. This bond was the most susceptible to being affected by the shearing mechanism, 
resulting in degradation by cleavage as proposed. Evidence shows that the shearing force generated by the rapid motion of the nano-knives was responsible for the breaking of these chemical bonds.

\subsection{Environmental significance}

$\mathrm{CMC}$ is a synthetic polymer compound that is widely used in various manufacturing processes for cosmetics, foods, medicines and paper making, amongst others (Bansal et al., 2010; Zorba and Ova, 1999). Industrial wastewater discharges with high CMC levels can result in a depletion of dissolved oxygen (DO) and cause serious harm to the environment, organisms and human health (Kortekaas et al., 1998). Such discharges can also restrain the growth of microorganisms due to the high viscosity that CMC presents even at low concentration (Lee et al., 2009). To date, a number of techniques have been developed to treat excess CMC concentrations in wastewater. For example, higher temperature promoted the thermal degradation of CMC (de Britto and Assis, 2009). However, thermal degradation of CMC can produce harmful small-molecule compounds such as ketones and aldehydes (Brebu et al., 2004; Golas et al., 2010). Another approach is using an anaerobic/aerobic bioreactor with symbiotic bacteria which degrade CMC in wastewater (Ji et al., 2013). However, any loss or imbalance of the three different cellulose enzymes would lead to a decrease in the cellulose degradation efficiency in an anaerobic/aerobic bio-reactor. Also, both thermal and ultrasonic approaches seem to be hindered by the large amount of heat or electric energy that is consumed during the degradation of CMC. In most cases, the majority of the energy carried by electron beam radiation is absorbed by 
water rather than $\mathrm{CMC}$ (Choi et al., 2008). Other approaches, such as those using $\gamma$ radiation degradation (Choi et al., 2008), present obvious issues for human health. Finally, oxidation-reduction degradation needs chemical reagents, which in turn can cause secondary pollution. Li et al. (2011a) reported that more $\mathrm{NaIO}_{4}$ or a net decrease in $\mathrm{pH}$ value would produce considerably more aldehydes as by-products. In this study, the nano-knife technology, which can cut the chemical bond and consequently decompose the $\mathrm{CMC}$ molecules in aqueous solution, is completely dependent on physical principles and easy to operate. The technique is relatively affordable and environment-friendly without secondary pollution, and provides a potential strategy for physical-based decomposition of recalcitrant organic pollutants from aqueous solution without the need for chemical or biological reagents.

\section{Conclusions}

The results from this study indicated how the use of a high-speed mechanical blade without quartz sand could rapidly decrease the viscosity of CMC solution by disaggregation of the network thread structure formed by the $\mathrm{CMC}$ macromolecules. Nevertheless, the mechanical shearing force seemed insufficient to break the long chain of CMC molecules in aqueous solution. The addition of quartz sand grains acting as nano-knives into the system allowed instead the scission of the weak bond ( $\beta$-1-4-glycosidic bond) in the CMC backbone to take place. The combined effect of the nano-knife material assisted by hydrodynamic forces seemed to be highly effective in promoting the physical degradation of $\mathrm{CMC}$ and therefore represents a very promising approach to be used as an alternative to conventional methods. 


\section{Acknowledgments}

The work was supported by the National Natural Science Foundation of China (Nos.

21277161, 41573114) and the National Key Research and Development Program of China (No. 2017YFA0207204). 


\section{References}

Bansal, P., Hall, M., Realff, M.J., Lee, J.H., Bommarius, A.S., 2010. Multivariate statistical analysis of X-ray data from cellulose: A new method to determine degree of crystallinity and predict hydrolysis rates. Bioresource Technol. 101, $4461-4471$.

Brebu, M., Bhaskar, T., Murai, K., Muto, A., Sakata, Y., Uddin, M.A., 2004. The effect of PVC and/or PET on thermal degradation of polymer mixtures containing brominated ABS. Fuel 83, 2021-2028.

Cao, Y., Li, H.L., Xu, Y., 1997. Studies on molecular morphology of carboxy methylcellulose based polymer surfactants in solution. Polym. Mat. Sci. Eng. 13, $106-110$.

Chen, Z., Zhang, W.J., Wang, D.S., Ma, T., Bai, R.Y., Yu, D.Z., 2016. Enhancement of waste activated sludge dewaterability using calcium peroxide pre-oxidation and chemical re-flocculation. Water Res. 103, 170-181.

Cheng, J., Vecitis, C.D., Park, H., Mader, B.T., Hoffmann, M.R., 2010. Sonochemical degradation of perfluorooctane sulfonate (PFOS) and perfluorooctanoate (PFOA) in groundwater: kinetic effects of matrix inorganics. Environ. Sci. Technol. 44, 445-450.

Choi, J.I., Lee, H.S., Kim, J.H., Lee, K.W., Lee, J.W., Seo, S.J., et al., 2008. Controlling the radiation degradation of carboxymethylcellulose solution. Polym. Degrad. Stabil. 93, 310-315. 
D’Almeida, A.R., Dias, M.L., 1997. Comparative study of shear degradation of carboxymethylcellulose and poly(ethylene oxide) in aqueous solution. Polym. Degrad. Stabil. 56, 331-337.

de Britto, D., Assis, O.B.G., 2009. Thermal degradation of carboxymethylcellulose in different salty forms. Thermochim Acta. 494, 115-122.

Gao, X.Y., Cao, Y., Song, X.F., Zhang, Z., Zhuang, X.L., He, C.L., et al., 2014. Biodegradable, pH-responsive carboxymethyl cellulose/poly(acrylic acid) hydrogels for oral insulin delivery. Macromol. Biosci. 14, 565-575.

Golas, P.L., Louie, S., Lowry, G.V., Matyjaszewski, K., Tilton, R.D., 2010. Comparative study of polymeric stabilizers for magnetite nanoparticles using ATRP. Langmuir 26, 16890-16900.

He, M., Chen, W., Dong, X., 2002. Polymer physics. Fudan University Press.

Ji, G.D., Wang, C., Guo, F., 2013. Characterisation of microbial floras and functional gene levels in an anaerobic/aerobic bio-reactor for the degradation of carboxymethyl cellulose. Appl. Microbiol. Biot. 97, 3195-3206.

Kortekaas, S., Wijngaarde, R.R., Klomp, J.W., Lettinga, G., Field, J.A., 1998. Anaerobic treatment of hemp thermomechanical pulping wastewater. Water Res. $32,3362-3370$.

Lee, H.S., Choi, J.I., Kim, J.H., Lee, K.W., Chung, Y.J., Shin, M.H., et al., 2009. Investigation on radiation degradation of carboxymethylcellulose by ionizing irradiation. Appl. Radiat. Isotopes 67, 1513-1515. 
Li, H.L., Wu, B., Mu, C.D., Lin, W., 2011a. Concomitant degradation in periodate oxidation of carboxymethyl cellulose. Carbohyd. Polym. 84, 881-886.

Li, J., Yu, Y.Q., Li, X.Y., Wang, W., Yu, G., Deng, S.B., et al., 2015. Maximizing carbon efficiency of petrochemical production from catalytic co-pyrolysis of biomass and plastics using gallium-containing MFI zeolites. Appl. Catal. B Environ. 172, 154-164.

Li, J.H., Li, J.L., Yang, N.W., 2011b. Experimental research on affects the viscosity of carboxymethylcelluloses. Appl. Chem. Ind. 40, 479-485.

Mohod, A.V., Gogate, P.R., 2011. Ultrasonic degradation of polymers: Effect of operating parameters and intensification using additives for carboxymethyl cellulose (CMC) and polyvinyl alcohol (PVA). Ultrason. Sonochem. 18, 727-734.

Ruzene, D.S., Goncalves, A.R., Teixeira, J.A., De Amorim, M.T.P., 2007. Carboxymethyl cellulose obtained by ethanol/water organosolv process under acid conditions. Appl. Biochem. Biotech. 137, 573-582.

Taghizadeh, M.T., Rad, H., Abdollahi, R., 2012. A Kinetic Study of Ultrasonic Degradation of Carboxymethyl Cellulose. J. Appl. Polym. Sci. 123, 1896-1904.

Yang, B., Jiang, C.J., Yu, G., Zhuo, Q.F., Deng, S.B., Wu, J.H., et al., 2015. Highly efficient electrochemical degradation of perfluorooctanoic acid (PFOA) by Fdoped Ti/SnO 2 electrode. J. Hazard. Mat. 299, 417-424.

Zhang, T.L., Pan, G., Zhou, Q., 2016. Temperature effect on photolysis decomposing of perfluorooctanoic acid. J. Environ. Sci. 42, 126-133. 
Zhang, W., Huang, J., Xu, F.Y., Deng, S.B., Zhu, W.P., Yu, G., 2011. Mechanochemical destruction of pentachloronitrobenzene with reactive iron powder. J. Hazard. Mater. 198, 275-281.

Zhang, W., Huang, J., Yu, G., Deng, S.B., Zhu, W.P., 2010. Mechanochemical destruction of dechlorane plus with calcium oxide. Chemosphere 81, 345-350.

Zorba, M., Ova, G., 1999. An improved method for the quantitative determination of carboxymethyl cellulose in food products. Food Hydrocolloid. 13, 73-76. 